\title{
Efeito de protocolo hormonal e programa de luz artificial na gestação de éguas receptoras de embrião durante o período de transição de primavera*
}

\section{Effect of hormonal protocol and artificial light program on the pregnancy of embryo recipient mares during spring transition period}

\author{
Guilherme Cunha de Oliveira, ${ }^{* *}$ David Horber, ${ }^{* * *}$ Amanda Pifano Neto Quintal, ${ }^{* * *}$ André Belico de Vasconcelos ${ }^{* * * *}$
}

\begin{abstract}
Resumo
O objetivo do estudo foi comparar o efeito das técnicas hormonais e de luz artificial nas éguas receptoras de embrião acíclicas avaliando as taxas de gestação aos 14 e 28 dias durante a fase de transição de primavera. Os 48 animais foram distribuídos aleatoriamente nos grupos: controle (CONT, n=16), éguas cíclicas na fase ovulatória; luz artificial (LUZ, n=16), éguas acíclicas submetidas ao tratamento de luz artificial; e hormônio (HORM, n=16), éguas acíclicas submetidas ao protocolo hormonal na fase de transição. As éguas do grupo LUZ foram estimuladas por 60 dias com luz artificial durante cinco horas por dia. Nos grupos CONT e LUZ, quando observada a presença de folículo $\geq 35 \mathrm{~mm}$ de diâmetro e edema uterino $\geq$ grau II, foram administrados 1,5 mg de acetato de deslorelina e 1500 UI de hCG para induzir a ovulação. As éguas do grupo HORM foram tratadas com três doses de 1,5 mg de benzoato de estradiol e seguiram os mesmos protocolos dos Grupos CONT e LUZ. Foi avaliada a taxa de gestação por ultrassonografia aos 14 dias e confirmação aos 28 em todos os grupos experimentais. Foi realizada análise descritiva e teste Qui-quadrado (significância de 5\%). Taxas de gestação aos 14 e 28 dias foram semelhantes ( $p>0,05)$ entre todos os grupos. Os tratamentos HORM e LUZ durante o período de transição inverno-primavera mostraram-se eficazes para atender ao programa de transferência de embrião. Por ser um método mais natural, o protocolo LUZ tem potencial como mais uma ferramenta biotecnológica na reprodução de equinos.
\end{abstract}

Palavras-chave: anestro; equino; sazonalidade reprodutiva.

\begin{abstract}
The aim of this study was to compare the effect of hormonal and artificial light techniques on acyclic embryo recipient mares by assessing pregnancy rates at 14 and 28 days during the spring transition period. The 48 animals were randomly assigned to the groups: control (CONT, $n=16$ ), cyclic mares in the ovulatory phase; artificial light (LIGHT, $n=16$ ), acyclic mares subjected to artificial light treatment; and hormone (HORM, $n=16)$, acyclic mares submitted to hormonal protocol in transition phase. In the LIGHT group, mares were stimulated with artificial light for five hours a day, for 60 days. In CONT and LIGHT groups, when a follicle $\geq 35$ $\mathrm{mm}$ in diameter and uterine edema $\geq$ grade II were observed, $1.5 \mathrm{mg}$ of deslorelin acetate and 1500 IU hCG were administered to induce ovulation. In the HORM group, mares were treated with three doses of $1.5 \mathrm{mg}$ of estradiol benzoate and followed the same protocols as the CONT and LIGHT groups. Pregnancy rate was assessed by ultrasound at 14 days and confirmation at 28 days in all experimental groups. Descriptive analysis and chi-square test ( $5 \%$ significance) were performed. Pregnancy rates at 14 and 28 days were similar ( $p>0.05$ ) among all groups. The HORM and LIGHT treatments during the winter-spring transition period proved to be effective during the embryo transfer programs. As it is a more natural method, the LIGHT protocol has the potential to be one more biotechnological tool in equine reproduction.
\end{abstract}

Keywords: anestrous; equine, reproductive seasonality.

\section{Introdução}

O aprimoramento da técnica de transferência de embrião (TE) em equinos nos últimos anos possibilitou o melhoramento genético das raças, como também dos seus cruzamentos. Por conseguinte, este avanço possibilita atualmente o desenvolvimento de estratégias com interações de técnicas que tem como objetivo melhorar as taxas de prenhez (Alvarenga e Tongu, 2017).

A espécie equina é classificada reprodutivamente como poliéstrica estacional de dias longos (Claes et al., 2019). O padrão sazonal das atividades reprodutivas na égua

\footnotetext{
${ }^{*}$ Recebido em 25 de novembro de 2019 e aceito em 29 de junho de 2020.

**Discente do Programa de Pós Graduação em Sanidade e Produção Animal nos Trópicos, UNIUBE.

*** Médico Veterinário na Equine Embryo Transfer Facility.

****Docente da Faculdade Associada de Uberaba, FAZU.

${ }^{\star * * * *}$ Docente do Programa de Pós-Graduação em Sanidade e Produção Animal nos Trópicos, UNIUBE.
} 
normalmente é decorrente da diminuição das ovulações no período de transição de outono, mínima ou ausente no inverno, aumentando gradativamente na primavera e chegando ao pico no verão. Este padrão sazonal é decorrente das variações dos níveis de hormônio liberador de gonadotrofina $(\mathrm{GnRH})$ (Guillaume et al., 2000; Conley, 2016; Murphy, 2019). Estas variações ocorrem devido ao sinal luminoso que é transmitido pelo trato retino-hipotalâmico ao pareado bilateralmente do núcleo supraquiasmático no hipotálamo, que codifica estes sinais em 'hora do dia', conduzindo as mudanças hormonais e fisiológicas (Murphy, 2019; O'Brien et al., 2020).

Devido aos efeitos da sazonalidade sobre a espécie equina e a complexidade de manejo das receptoras, médicos veterinários e criadores tem procurado aplicar protocolos hormonais como uma estratégia para o aumento da taxa de concepção (Alvarenga e Tongu, 2017). Assim, uma das formas para minimizar a baixa taxa de concepção é ajustar o protocolo de TE, afim de que as atividades ovarianas das éguas doadoras ocorram mais cedo, e as éguas receptoras mantenham o anestro sazonal (Silva et al., 2017; Roser et al., 2019).

Estudos buscam antecipar a sazonalidade reprodutiva das éguas utilizando-as como receptoras acíclicas, para tal utilizam o tratamento com luz artificial iniciando o protocolo a partir do solstício de inverno (Guillaume et al., 2000; Claes et al., 2019; Roser et al., 2019). A exposição a luz natural somada a luz incandescente por um período maior ou igual a 16 horas mimetiza o solstício de verão, período de reprodução das equinos (Guillaume et al., 2000). Por outro lado, várias pesquisas relatam a eficiência de protocolos hormonais utilizando estrógeno (E2) e progesterona ( $\mathrm{P} 4$ ) em éguas acíclicas receptoras de embriões. A utilização desses hormônios possibilita a indução de mudanças fisiológicas desejáveis no útero, como edema endometrial, aumento do tônus uterino e estímulo à secreção histotrófica, similares as que ocorrem em éguas cíclicas (Kaercher et al., 2013; Coffman et al., 2014; Botelho et al., 2015; Silva et al., 2016).

Assim o objetivo deste estudo foi comparar o efeito da técnica hormonal e de luz artificial nas éguas receptoras de embrião acíclicas avaliando as taxas de gestação aos 14 e 28 dias durante a fase de transição de primavera.

\section{Material e métodos}

O projeto foi aprovado pelo Comitê de Ética em Experimentação Animal da Universidade de Uberaba (CEEA nº 04512019).

O estudo foi realizado em uma central especializada em reprodução equina (Equine Embryo Transfer Facility), durante os meses de abril a setembro de 2019, na cidade de Weatherford, Texas, EUA (latitude $32^{\circ} \mathrm{N}$ e longitude $97^{\circ} \mathrm{O}$ ).

Foram utilizadas 48 éguas mestiças, multíparas, com idade entre quatro e 12 anos, sem histórico reprodutivo de patologias, com peso médio de $455 \mathrm{~kg}$ e escore de condição corporal de 5 a 7 (Henneke et al. 1983).

Os animais foram mantidos em piquetes sob o mesmo manejo alimentar, com grama Bermuda (Cynodon dactylon) e Alfafa (Medicago sativa), arraçoadas com concentrado de $12 \%$ de proteína bruta uma vez ao dia e livre acesso à água em bebedouro coletivo e sal mineralizado.
Após avaliação clínica veterinária por palpação e ultrassonografia transretal, foram selecionadas para estudo apenas éguas ginecologicamente sadias, sendo ainda separadas as éguas cíclicas e acíclicas para sorteio aleatório dos grupos experimentais.

As 48 éguas selecionadas foram distribuídas em: grupo controle (CONT, $n=16$ ) composto por éguas cíclicas com ciclos estrais regulares normais e presença de folículo ovulatório e/ ou corpo lúteo (CL); grupo luz artificial (LUZ, $n=16)$ composto por éguas acíclicas submetidas ao tratamento de luz artificial com folículos com diâmetro inferior a $20 \mathrm{~mm}$ e ausência de $\mathrm{CL}$; e grupo hormônio (HORM, $n=16$ ) composto por éguas acíclicas submetidas ao protocolo hormonal na fase de transição, apresentando características ovarianas semelhantes ao grupo LUZ.

Todas as éguas foram examinadas a cada 24 horas por meio de ultrassonografia transretal modo-B (Hitachi, ALOKA Prosound 2, Japão) equipado com transdutor linear de $5.0 \mathrm{MHz}$ de frequência. O exame ultrassonográfico foi utilizado para avaliação da condição uterina e ovariana das receptoras assim como para o diagnóstico de gestação aos 14 e 28 dias.

As éguas do grupo LUZ foram estimuladas por 60 dias de luz artificial, nos meses de abril e maio (primavera). Para tal foram distribuídos igualmente em um piquete, holofotes a uma intensidade de 100 lux de luz incandescente fria. Os holofotes permaneceram acesos durante cinco horas por dia (17:00 às 22:00 h), utilizando-se um temporizador analógico (Exatron ${ }^{\circledR}$, Canoas, Brasil), diretamente ligado ao sistema de iluminação do piquete. Após o período de exposição à luz artificial, os animais retornavam para o piquete onde se encontravam os demais animais do experimento.

As éguas cíclicas, pertencentes aos grupos CONT e LUZ com folículo $\geq 35 \mathrm{~mm}$ de diâmetro e edema uterino $\geq$ grau II, receberam 1,5 mg de acetato de deslorelina (Equine Embryo Transfer Facility, Weatherford, Texas, USA), por via intramuscular e 1500 UI de hCG (Equine Embryo Transfer Facility, Weatherford, Texas, USA), por via intravenosa para induzir a ovulação. Esses animais foram novamente avaliados a cada 24 horas para verificar o dia da ovulação (D0).

As éguas do grupo HORM, receberam tratamento com de benzoato de estradiol (Equine Embryo Transfer Facility, Weatherford, Texas, USA), por via intramuscular, durante três dias (Silva et al., 2019). Vinte e quatro horas após a terceira aplicação de estradiol, as éguas foram examinadas por meio de ultrassonografia para avaliação do edema uterino. Confirmada a presença de edema uterino $\geq$ grau II (Gonçalves et al., 2020), estas foram consideradas aptas para administração de 180 mg de P4 de curta ação (Equine Embryo Transfer Facility, Weatherford, Texas, USA), por via intramuscular em um único ponto, sendo esse dia considerado o D0, ou seja, o mesmo utilizado para os grupos CONT e LUZ.

As receptoras foram sincronizadas com as doadoras para a TE entre quatro a sete dias após a ovulação (D4 a D7). Previamente as receptoras foram classificadas de acordo com o tônus uterino, ecogenicidade endometrial e presença de CL, somente nas éguas cíclicas, que posteriormente foram ordenadas pela qualidade uterina para recepção do embrião (Kaercher et al., 2013). 
Os embriões eram oriundos de programas comerciais de TE de todo o estado do Texas. Todos os embriões utilizados no experimento foram considerados homogêneos com blastômero de tamanho e cor uniformes, conforme as recomendações da IETS (International Embryo Transfer Society), com grau de qualidade 1 e 2 (McKinnon. Squires Carnevale, 1988).

A técnica de transferência utilizada no presente trabalho foi a não cirúrgica coberta, como relatado por Fleury e colaboradores (2007) e os diagnósticos de gestação foram realizados em dois momentos, 14 e 28 dias após a data de ovulação da doadora identificada com a utilização de exame ultrassonográfico. Foram consideradas gestantes as éguas onde se verificou a presença de vesícula embrionária no útero, assim como a presença de $\mathrm{CL}$ compacto e uniformemente ecogênico no ovário.

Após a confirmação do diagnóstico de gestação, as receptoras do grupo HORM permaneceram com a administração de P4 injetável (1500 mg) (Silva et al., 2016; Silva et al., 2019). Conforme descrito no esquema experimental (Figura1).

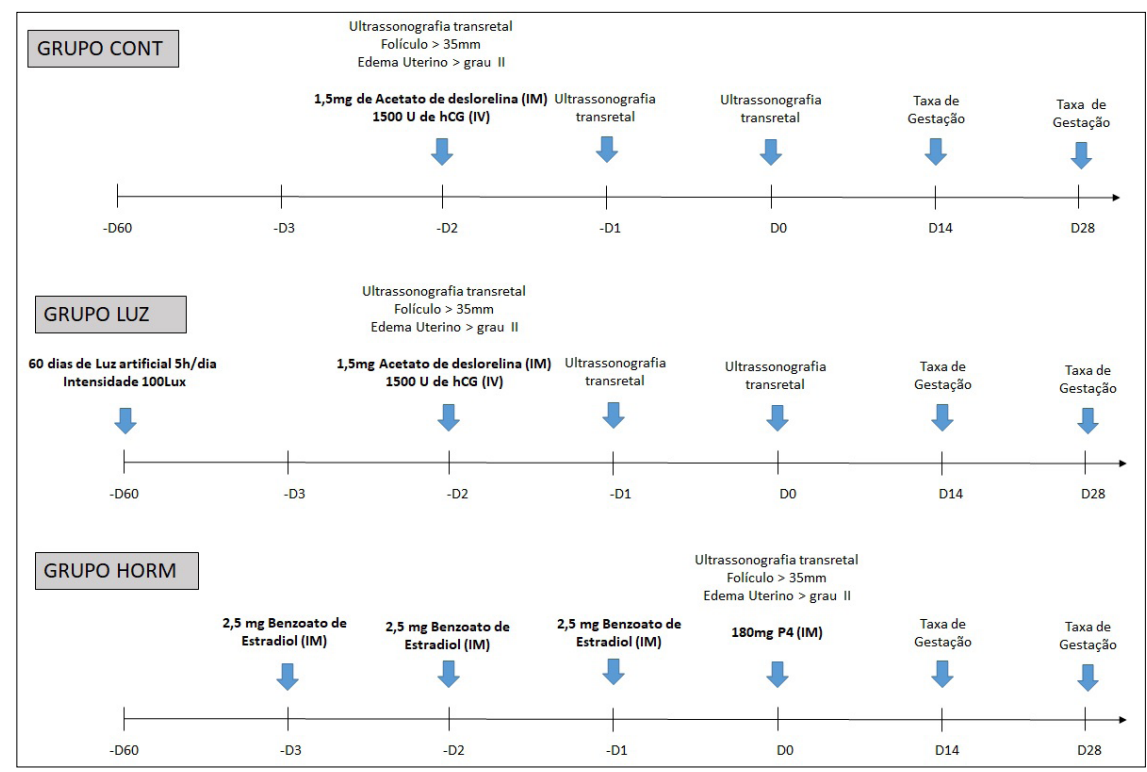

Figura 1: Descrição esquemática dos procedimentos utilizados em cada grupo experimental. CONT (Controle, éguas cíclicas); LUZ (Luz artificial, éguas acíclicas) e HORM (Protocolo hormonal, éguas acíclicas).

Para análise estatística foi utilizado o programa GraphPadPrism 6.0 (GraphPad Software Inc., San Diego, CA, USA). O modelo matemático foi o delineamento de blocos casualizados (DBC), aplicando análise descritiva em percentual, seguida do teste de qui-quadrado, ao nível de significância de $5 \%(p<0,05)$.

\section{Resultado e Discussão}

Não houve diferença $(p>0,05)$ entre os grupos CONT, LUZ e HORM nas taxas de gestação aos 14 dias $(87,5 \%$; $93,8 \%$ e $87,5 \%$ ) e aos 28 dias ( $81,3 \% ; 87,5 \%$ e $81,3 \%$ ), respectivamente.

As taxas de gestação de éguas cíclicas (controle) e acíclicas submetidas a protocolo hormonal também não apresentaram diferença de acordo com Kaercher et al. (2013), Silva et al (2016), Silva et al. (2017), Oliveira-Neto et al. (2018).
A taxa de gestação aos 28 dias no grupo CONT $(81,3 \%)$ foi superior aos valores maiores relatados na literatura, como $44,12 \%$ (Greco et al., 2012) e $75 \%$ (Oliveira Neto et al., 2018). Os resultados apontam a qualidade dos procedimentos de avaliação e seleção das éguas submetidas no presente experimento. Os valores da taxa de gestação reforçam os critérios e o procedimento da avaliação ginecológica realizada nas receptoras, antes e no momento da inovulação (Snider, 2015; Weber et al., 2018).

Éguas em período de transição de primavera apresentam atividade ovariana e concentrações de LH (hormônio luteinizante) menores do que na fase ovulatória e consequentemente, acarretam baixas concentrações de hormônios endógenos (Snider, 2015; Max et al., 2017; Silva et al., 2019). Isto torna justificável a utilização de protocolos hormonais à base de E2 e P4 em períodos transicionais, obtendo índices de gestação similares a éguas cíclicas em programas comerciais de TE (Botelho et al., 2015; Silva et al., 2017; Oliveira Neto et al., 2018).

Desta forma provavelmente as taxas de gestação obtidas no grupo HORM, no presente experimento estão relacionadas ao uso exógeno dos hormônios E2 e P4, que possibilitaram mudanças no útero, semelhantes às que ocorrem nas éguas cíclicas. Esses hormônios (naturais ou sintéticos) induzem resposta uterina semelhante às alterações que ocorrem em éguas cíclicas, como: edema endometrial seguido de aumento dos tônus uterino e estímulo à secreção histotrófica (Silva et al., 2016). O E2 promove a formação de edema uterino similar ao estro e, posteriormente, a administração da P4 promove aumento do tônus uterinos equivalente ao observado no diestro, preparando-o para receber o embrião (Silva et al., 2016; Silva et al., 2017; Roser et al., 2019).

Diversas concentrações de BE são utilizadas para propiciar a condição uterina ideal nas éguas. Neste estudo, para o grupo de éguas acíclicas, foram utilizadas três doses $\mathrm{BE}(2,5$ $\mathrm{mg}$ ). Protocolo semelhante foi realizado por Oliveira Neto et al. (2018), que utilizou três doses nos dias -3 (10 mg), -2 (20 mg) e -1 (10 $\mathrm{mg}$ ) de BE resultando em maior expressão do receptor de P4 no trato reprodutivo. Contrapondo o protocolo descrito por Silva e colaboradores (2017) que propuseram o uso de uma única dose de $2,5 \mathrm{mg}$ de BE seguido de $1500 \mathrm{mg}$ de $\mathrm{P} 4$ de longa ação no D0 em éguas acíclicas. Como também o protocolo de Kaercher et al. (2013) que relataram o tratamento de receptoras acíclicas com uma única dose de $\mathrm{BE}$ seguido de $400 \mathrm{mg}$ de $\mathrm{P} 4$ de longa ação administrada semanalmente.

As éguas do grupo LUZ e do grupo CONT receberam o análogo ao GnRH (acetato de deslorelina), de forma a melhorar a eficiência reprodutiva, reduzindo o período do estro e sincronizando o ciclo do animal, visto que a deslorelina estimula a liberação prolongada de hormônio luteinizante (LH) e (hormônio folículo estimulante) FSH da pituitária anterior. 
A utilização de hormônios sintéticos tem sua relevância para o programa de transferência de embriões, contudo observa-se que o grupo LUZ, embora não se tenha encontrado significância, apresentou percentuais maiores de taxa de gestação quando comparado ao grupo CONT e HORM.

A égua é um animal poliéstrico, anual ou estacional, cujas atividades ovarianas máximas ocorrem durante o período de primavera-verão. Sabe-se que a luminosidade em animais que apresentam fotoperíodo positivo leva a inibição na produção de melatonina pela glândula pineal, permitindo a produção de GnRH, o que leva a liberação de LH e FSH (Conley, 2016). Assim, especula-se no presente estudo, que o tratamento de luz artificial, pode ter induzido uma maior liberação de $\mathrm{GnRH}$, e que ao ser administrado acetato de deslorelina, potencializou a janela de reprodução das éguas, favorecendo o período de transferência do embrião.

De acordo com Guillaume et al. (2000), o protocolo com luz artificial é a forma efetiva para que se consiga a primeira ovulação do período de transição. Este fato foi também observado no presente estudo, uma vez que as taxas de gestação dos protocolos CONT, LUZ e HORM foram semelhantes.

\section{Conclusão}

Concluímos que éguas na fase de transição inverno-primavera quando submetidas ao protocolo de luz artificial podem ser utilizadas no programa de transferência de embriões, com resultados satisfatórios semelhantes aos tratamentos que utilizam indução hormonal.

\section{Agradecimentos}

A Coordenação de Aperfeiçoamento de Pessoal de Nível Superior - Brasil (CAPES) pela concessão de bolsa PROSUP/TAXA durante o programa de mestrado - Código de Financiamento 001.

\section{Referências}

ALVARENGA, M.A.; TONGU, E.A.O. Estratégias para melhorar a eficiência reprodutiva em programas de transferência de embrião de equinos. Revista Brasileira Reprodução Animal, v.41, n.1, p.19-24, 2017.

BOTELHO, J.H.V.; PESSOA, G.O.; ROCHA, L.G.P.; YESTE, M. Hormone supplementation protocol using estradiol benzoate and long-acting progesterone is efficient in maintaining pregnancy of anovulatory recipient mares during autumn transitional phase. Animal Reproduction Science, v. 153, p. 39 - 43, 2015.

CLAES, A.; CUERVO-ARANGO, J.; VAN DEN BROEK, J.; GALLI, C.; COLLEONI, S.; LAZZARI, G.; DEELEN, C.; BEITSMA, M.; STOUT, T.A. Factors affecting the likelihood of pregnancy and embryonic loss after transfer of cryopreserved in vitro produced equine embryos. Equine, v. 51, n. 4, p. 446-450, 2019. COFFMAN, E.A.; PINTO, C.R.; SNYDER, H.K.; LEISINGER, C.A.; COLE, K.; WHISNANT, C.S. Antiluteogenic effects of serial prostaglandin $\mathrm{F} 2 \alpha$ administration in cycling mares. Theriogenology, v. 82, p. 1241 - 1245, 2014.

CONLEY, A.J. Review of the reproductive endocrinology of the pregnant and parturient mare. Theriogenology, v. 86, p. $355-65$, 2016.

FLEURY, P.D.C.; ALONSO M.A.; SOUSA F.A.C.; ANDRADE A.F.C.; ARRUDA R.P. Uso da gonadotrofina coriônica humana (hCG) visando melhorar as características reprodutivas e fertilidade de receptoras de embriões eqüinos. Revista Brasileira Reprodução Animal, v.31, p.27-31, 2007.

GONÇALVES, G.R.; MOROTTI, F.; COLOMBO, AHB.; BONATO, DV.; BIZARRO-SILVA, C.; ROSA, CO.; CAVALIERI,

FLB.; SENEDA, MM. Influence of age and ovarian antral follicle count on the reproductive characteristics of embryo donor mares. Veterinary Record Published Online, 2020. doi: 10.1136/vr.105526

GRECO, G.M.; BURLAMAQUI, F.L.G.; PINNA, A.E.; QUEIROZ, F.J.R.; CUNHA, M.P.S.;BRANDÃO, F.Z. . Revista Brasileira de Zootecnia, v.41, n.3, p.607-611, 2012.

GUILLAUME, D.; DUCHAMP, G.; NAGY, P.; PALMER, $E$. Determination of minimun light treatment required for photoestimulation of winter anoestrous mares. Journal of Reproduction and Fertility, v. 56, p. 205 - 216, 2000.
HENNEKE, D. R.; POTTER, G. D.; KREIDER, J. L.; YEATES, B. F. Relationship between condition score, physical measurements and body fat percentage in mares. Equine Veterinary Journal. v. 15, n. 4, p. $371-372,1983$.

KAERCHER, F.; KOZICKI, L.E.; CAMARGO, C.E.; WEISS, R.R.; SANTOS, I.W.; MURADAS, P.R.; BERTOL, M.A.F.; ABREU, R.A. Embryo Transfer in Anovulatory Recipient Mares Treated with Estradiol Benzoate and Long-Acting Progesterone. Journal of Equine Veterinary Science, v. 33, p. 205 - 209, 2013.

MAX, M.C.; SILVA, C.B.; GONZÁLEZ, S.M.; LINDQUIST, A.G.; BÚFALO, I; GOMES, R.G.; MOROTTI, F.; COSTA, C.B.; BARREIROS, T.; LISBOA, L.A.; SENEDA, M.M. The development and integrity of equine pre-antral follicles cultured in vitro with follicle-stimulating hormone $(\mathrm{FSH})$ supplementation. Reproduction in Domestical Animals, v. 52, n. 5, p. 899 - 904, 2017.

MCKINNON, A.O.; SQUIRES, E.L.; CARNEVALE, E.M. Ovariectomized steroid-treated mares as embryo transfer recipients and as a model to study the role of progestins in pregnancy maintenance. Theriogenology, v.29, p.1055-1063, 1988.

MURPHY, B.A. Circadian and circannual regulation in the horse: Internal timing in an elite athlete. Journal of Equine Veterinary Science, v. 76, p. $14-24,2019$.

OLIVEIRA NETO, I.V.; CANISSO, I.F.; SEGABINAZZI, L.G.; DELL'AQUA, C.P.F.; ALVARENGA, M.A.; PAPA, F.O.; DELL'AQUA JR, J.A. Synchronization of cyclic and acyclic embryo recipient mares with donor mares. Animal Reproduction Science, v. 190, p. $1-9,2018$.

O'BRIEN, C.; DARCY-DUNNE, M.R.; MURPHY, B.A. The effects of extended photoperiod and warmth on hair growth in ponies and horses at different times of year. PLoS One, v.15, n.1, e0227115, 2020.

ROSER, J.F.; ETCHARREN, M.V.; MIRAGAYA, M.H.; MUTTO, A.; COLGIN, M.; LOSINNO, L.; ROSS, P.J. Superovulation, embryo recovery, and pregnancy rates from seasonally anovulatory donor mares treated with recombinant equine FSH (reFSH). Theriogenology, v. 15, p. 291 - 295, 2019. 
SILVA,E.S.M.; LEITE, R.O.; MACIEL, L.F.S.; FRANCIA, C.C.D.A.; PADOVANI, C.R.; OLIVEIRA-FILHO, J.P.; MEIRA, C. Endometrial histomorphometry of anestrous mares under the influence of different embryo transfer hormonal protocols. Journal of Equine Veterinary Science, v. 81, p. 1-7, 2019. https://doi. org/10.1016/j.jevs.2019.102792.

SILVA, E.S.M.; IGNÁCIO, F.S.; FRITSCH, S.C.; ZANONI, D.S.; PANTOJA, J.C.F.; OLIVEIRA-FILHO, J.P.; MEIRA, C. Administration of $2.5 \mathrm{mg}$ of estradiol followed by $1,500 \mathrm{mg}$ of progesterone to anovulatory mares promote similar uterine morphology, hormone concentrations and molecular dynamics to those observed in cyclic mares. Theriogenology, v. 97, p. $159-$ 169, 2017.
SILVA, E.S.M.; ROSER, J.F.; GOMES, A.R.C.; FRITSCH, S.C.; PANTOJA, J.C.F.; OLIVEIRA FILHO, J.P.; MEIRA, C. Comparison of different regimens of estradiol benzoate treatments followed by long-acting progesterone to prepare noncycling mares as embryo recipients. Theriogenology, v. 86, n. 7, p. 1 - 8, 2016. DOI: 10.1016/j.theriogenology.2016.05.041.

SNIDER, T.A. Reproductive Disorders in Horses. Veterinary Clinics of North America: Equine Practice Journal, v. 31, n. 2, p. $389-405,2015$.

WEBER, R.; HOSPES, R.; WEHREND, A. Causes of abortion in horses - overview of the literature and own evaluations. Tierarztl Prax Ausg Grosstiere Nutztiere, v. 46, n. 1, p. 35 - 42, 2018. 